\title{
A chemical sensor based on a micromechanical cantilever array for the identification of gases and vapors
} \author{
T. Mezzacasa ${ }^{3}$, L. Scandella ${ }^{3}$, H.-J. Güntherodt ${ }^{2}$, Ch. Gerber ${ }^{1}$, J.K. Gimzewski ${ }^{1}$ \\ ${ }^{1}$ IBM Research Division, Zurich Research Laboratory, Säumerstrasse 4, CH-8803 Rüschlikon, Switzerland \\ ${ }^{2}$ Institute of Physics, University of Basel, Klingelbergstrasse 82, CH-4056 Basel, Switzerland \\ ${ }^{3}$ Paul-Scherrer-Institute, Laboratory for Micro- and Nanostructures, CH-5232 Villigen-PSI, Switzerland
}

H.P. Lang ${ }^{1,2, *}$, R. Berger ${ }^{1,2}$, F. Battiston ${ }^{2}$, J.-P. Ramseyer ${ }^{2}$, E. Meyer ${ }^{2}$, C. Andreoli ${ }^{1}$, J. Brugger ${ }^{1}$, P. Vettiger ${ }^{1}$, M. Despont ${ }^{1}$,

Received: 25 July 1997/Accepted: 1 October 1997

\begin{abstract}
We have built and operated a novel setup for the characterization and identification of gases or vapors based on sequential position readout via a beam-deflection technique from a microfabricated array of eight cantilever-type sensors. Each of the cantilevers can be coated on one side with a different sensor material to detect specific chemical interactions. We demonstrate that disturbances from vibrations and turbulent gas flow can be effectively removed in array sensors by taking difference signals with reference cantilevers. For example, $\mathrm{H}_{2}$ can be detected by its adsorption on a Pt-coated sensor because a change in surface stress causes a static bending of the sensor. The diffusion of various alcohols into polymethylmethacrylate induces resonance frequency shifts in a dynamic measuring mode and bending in the static mode, which allows one to distinguish between the various alcohols.
\end{abstract}

Sensor devices for detection of gases and vapors via specific coatings are gradually gaining importance in chemistry, materials science, and biochemistry owing to the increasing demand for detection of analytes at monolayer coverage. A field of increasing interest is the construction of so-called "electronic noses" capable of discerning different odors via a typical response pattern of the receptor layers to an analyte. Most devices currently applied involve square centimetersized detection areas and comparatively large gas volumes (typically $50-1000 \mathrm{~cm}^{3}$ ) resulting in relatively long response and purge times (typically seconds to minutes). The objective of the approach presented here is to use a small-volume analyte chamber $\left(11 \mathrm{~cm}^{3}\right)$ and miniaturized micromachined $\mathrm{Si}$ sensor arrays to improve sensitivity and reduce response time.

Parallelization in the field of scanning force microscopy (SFM) has been demonstrated by several groups whose work focused on parallel imaging [1-3] and lithography [4]. The use of SFM cantilevers as sensors was pioneered by Gimzewski et al. [5] by utilizing the bimaterial effect to develop a micromechanical calorimeter. This device allows the study of phase transitions, e.g., in picoliter volumes of

\footnotetext{
${ }^{*}$ Corresponding author
}

alkanes [6]. Further applications include photothermal spectroscopy [7], surface stress detection [8-10], and infrared detectors [11]. Seamless integration of such sensors in arrays allows the number to be increased and their versatility to be enhanced. The development of chemical sensor arrays is in progress $[12,13]$. The focus of our work is to downscale sensor arrays by applying micromechanical cantilever arrays as sensors. Such devices involve a very small surface area (typically $500 \times 100 \mu \mathrm{m}^{2}$ ), which facilitates a high-speed response (typically in the range of a few milliseconds [9]). Such sensor arrays can be employed as highly integrated, multipurpose and specific (bio-)chemical sensors, and can be regarded as a chemical "nose" with the capability to measure difference signals.

\section{Experimental}

A micromechanical array of currently eight Si cantilevers is placed in an analysis chamber into which various gases and vapors can be introduced. Our device can be operated in various media such as ambient air, vacuum, and liquids, which allows the characterization of gaseous analytes and liquids in chemistry and biochemistry. The position of the cantilever sensors is read out quasi-simultaneously (see Fig. 1) by a beam deflection technique using a fiber-optical ribbon of eight multimode fibers (Europtics Ltd, Whiston, Merseyside, UK), core diameter $62.5 \mu \mathrm{m}$ and MT8/ST connectors (NTT Corporation, Japan) at a pitch of $250 \mu \mathrm{m}$, which is identical to the spacing between individual cantilevers within the array to facilitate self-alignment. Eight light sources (HewlettPackard HLMP-8103, ultrabright light emitting diode, dominant wavelength $\lambda=644 \mathrm{~nm}$, typical intensity at $20 \mathrm{~mA} 3 \mathrm{~cd}$, typical radiant intensity at $20 \mathrm{~mA} 35.3 \mathrm{~mW} / \mathrm{sr}$ ) are used to illuminate the apex of each cantilever individually with a light beam through one of the fibers. The light reflected from each cantilever shines on a position-sensitive detector (twodimensional PSD 2L4, Laser Components, Olching/Munich, Germany). From the position of the spot on the PSD, the bending of each sensor is obtained. After converting the photocurrents into voltages, and then amplifying and digitizing 


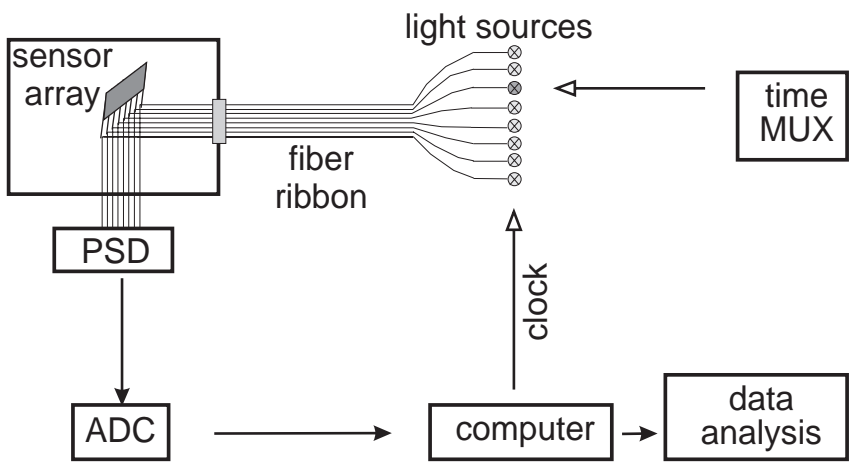

Fig. 1. Schematic setup of the chemical "nose" device illustrating the readout principle via optical beam deflection. Quasi-simultaneous readout of eight sensors is achieved by time-multiplexing eight light sources guided by an optical-fiber ribbon onto the sensor array located in the analysis chamber. The reflected light from the sensor's surface is collected by a positionsensitive detector (PSD), fed into an analog-to-digital converter (ADC) and stored in a computer for further analysis. The computer also generates the clock pulse for time-multiplexing (MUX)

the voltages, the cantilever bending inferred from the deflection signal is stored in computer memory for on-line data display and analysis. The temporal evolution of the bending of each sensor can be acquired by time-multiplexing the sensors (quasi-simultaneous readout by switching on and off the light sources).

Two types of sensor arrays (pitch $250 \mu \mathrm{m}$ ) were used (Fig. 2). Type A cantilevers are $500 \mu \mathrm{m}$ long, $100 \mu \mathrm{m}$ wide,

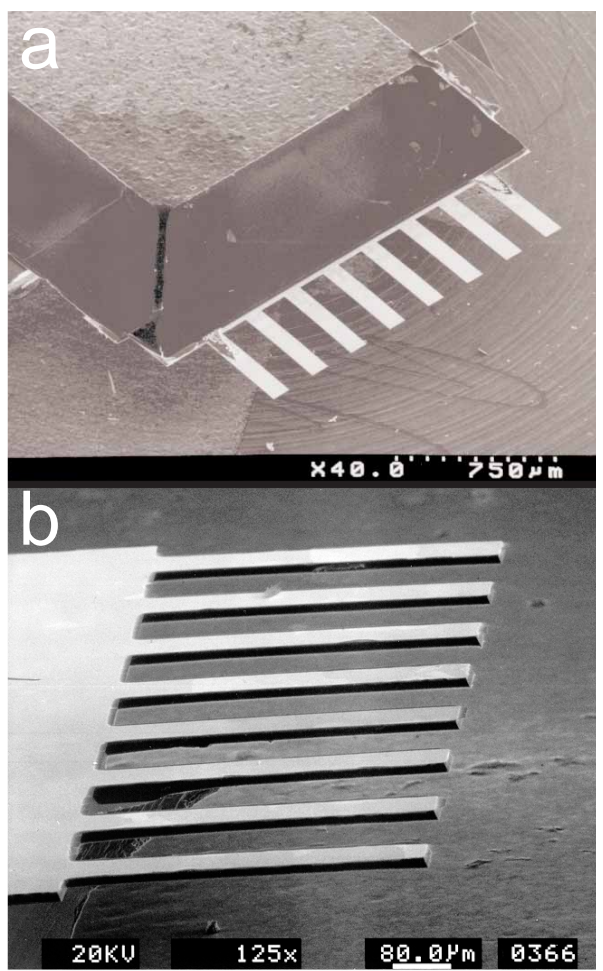

Fig. 2. a Scanning electron microscopy image of a type A micromechanical sensor array with eight sensors $(500 \mu \mathrm{m}$ long, $100 \mu \mathrm{m}$ wide and $0.8 \mu \mathrm{m}$ thick). The pitch is $250 \mu \mathrm{m}$. Such arrays are suitable for parallel and differential readout in the static measuring mode. b Type B sensor array with the same length and width as above, but a thickness of $12 \mu \mathrm{m}$. The greater stiffness of the sensors is suitable for the dynamic measuring mode and $0.8 \mu \mathrm{m}$ thick, and are made of epitaxially grown silicon on $\mathrm{Si}(100)$ (Fig. 2a). (Cantilever sensor arrays prepared at Micromechanics department, IBM Zurich Research Laboratory. Typical spring constant $0.02 \mathrm{~N} / \mathrm{m}$; typical resonance frequency $4 \mathrm{kHz}$.) Type B cantilevers have the same dimensions except for a thickness of $12 \mu \mathrm{m}$, and are made of $\mathrm{Si}(100)$ (Fig. 2b). (Cantilever sensor arrays prepared at Laboratory for Micro- and Nanostructures, Paul-Scherrer-Institute. Typical spring constant $1 \mathrm{~N} / \mathrm{m}$; typical resonance frequency $61 \mathrm{kHz}$.)

Sensor arrays of type A are applied to measure static bending upon exposure to gases and vapors. The bending is related to the change of surface stress $\sigma$ according to Stoney's formula [14]

$\sigma=\frac{E t^{2}}{6 R(1-v)}$

where $E$ is Young's modulus, $v$ is Poisson's ratio of the sensor material, $R$ the radius of curvature and $t$ the sensor thickness. The following material constants were used: $E=1.7 \times$ $10^{11} \mathrm{Nm}^{-2}, v=0.25$.

The radius of curvature is calculated from the cantilever length $l$ and the sensor deflection $\Delta z$ from

$R^{-1} \approx \frac{3 \Delta z}{2 l^{2}}$

Type B sensor arrays are suitable for use in the dynamic mode to observe changes of mass due to adsorption, similar to the operating principle of a quartz microbalance. The mass change $\Delta m$ derived from the resonance frequency shift is calculated from the following formula (cantilever uniformly loaded on one side) [15],

$\Delta m=\frac{k}{0.72 \pi^{2}}\left(\frac{1}{f_{\mathrm{f}}^{2}}-\frac{1}{f_{\mathrm{i}}^{2}}\right)$,

where $k$ is the spring constant, $f_{\mathrm{i}}$ the resonance frequency before and $f_{\mathrm{f}}$ the resonance frequency during the measurement.

As a receptor layer for a type A sensor, an electron-beamevaporated Pt layer was employed. Its thickness is $\approx 30 \mathrm{~nm}$ (including a 2-nm adherence layer of Ti). Appropriate coatings for the type $\mathrm{B}$ sensors involve polymer layers with a thickness of a few micrometers (such as polymethylmethacrylate, PMMA). All experiments presented here were performed in ambient air.

\section{Results and discussion}

\section{$2.1 \mathrm{H}_{2}$ detection in the static measuring mode}

$\mathrm{H}_{2}$ is known to chemisorb selectively on Pt surfaces [16]. Operation of the sensor at various temperatures and pressures would affect the adsorption rates of $\mathrm{H}_{2}$ on $\mathrm{Pt}$, and hence change bending and resonance frequency shift behavior. In order to study the formation of surface stress change during $\mathrm{H}_{2}$ adsorption on $\mathrm{Pt}$ at room temperature, four sensors of a type A cantilever array were coated on one side with a 30-nm electron-beam-evaporated Pt layer at their ends (s1s4), and four sensors were left uncoated ( $\mathrm{r} 1-\mathrm{r} 4)$ for reference.

$\mathrm{H}_{2}$ is introduced into the side of the chamber through a needle valve at an angle of $90^{\circ}$ with respect to the coated 

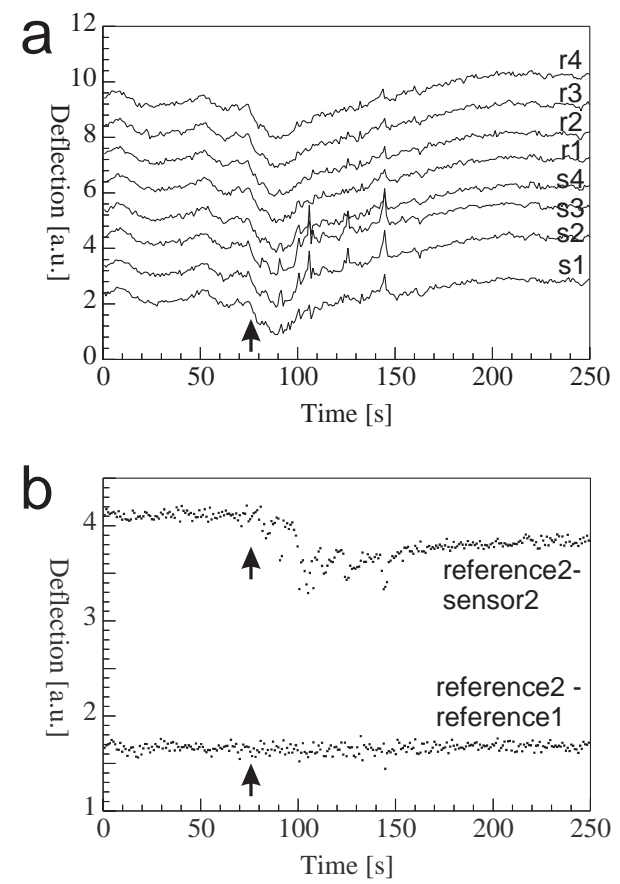

Fig. 3. a Quasi-simultaneously acquired responses (raw data) of eight individual sensors in a noisy environment. Four sensors are uncoated (reference sensors r1-r4) and four sensors are coated with a 30-nm layer of Pt (sensors s1-s4). At first glance, all these curves look similar because motion of the sensor array as a whole in the noisy environment is dominant. One deflection unit corresponds to $5 \mu \mathrm{m}$. b Evaluating the differences between the responses of a Pt-coated sensor and an uncoated reference sensor yields a characteristic signature of the chemisorption of $\mathrm{H}_{2}$ onto Pt. This changes the surface stress and causes the cantilever to bend (reference 2 - sensor2). The peaks in the reaction curve are believed to originate from sudden surface stress changes during the chemisorption process. The difference between two reference sensors (reference 2 - reference1) does not yield a net signal. The arrow indicates the insertion of $\mathrm{H}_{2}$ into the analysis chamber. One deflection unit corresponds to $5 \mu \mathrm{m}$ or a surface stress change of $0.73 \mathrm{Nm}^{-1}$

surface of the cantilevers. The flow rate is $1 \mathrm{mbar} \ell \mathrm{s}^{-1}$. This surface stress change on $\mathrm{H}_{2}$ adsorption causes the sensor to bend. Figure 3 a shows the recorded deflection signals of the eight cantilevers. At first glance, all cantilevers seem to undergo the same motion, owing to superimposed noise. The major advantage of this device over a single sensor is its capability to use some cantilevers within the array as a reference. By calculating the difference between the responses of a coated and an uncoated sensor (see Fig. 3b), we obtain the $\mathrm{H}_{2}$ adsorption reaction response. The curve shown in the upper part of Fig. 3b is typical of the adsorption of $\mathrm{H}_{2}$ on $\mathrm{Pt}$ and provides a signature (time scale, curve shape, and slope) for analyte recognition, e.g., by a neural network when several different sensors are used. Quantification of analyte concentrations is possible by utilizing the magnitude of the signal. The other difference signals have a very similar curve shape. Small differences in magnitude are due to inhomogeneity of the sensor coatings. No such signature is observed in the response difference of two uncoated reference cantilevers (lower part of Fig. 3b). Exposing a different gas (e.g., $\mathrm{CO}_{2}$ ) to the Pt receptor layer does not yield a response either. Utilizing such characteristic signatures, the device operates like a chemical "nose" for gases and vapors. The micromechanical design of the sensor array provides a small receptor area that allows short response times and high sensitivity. The
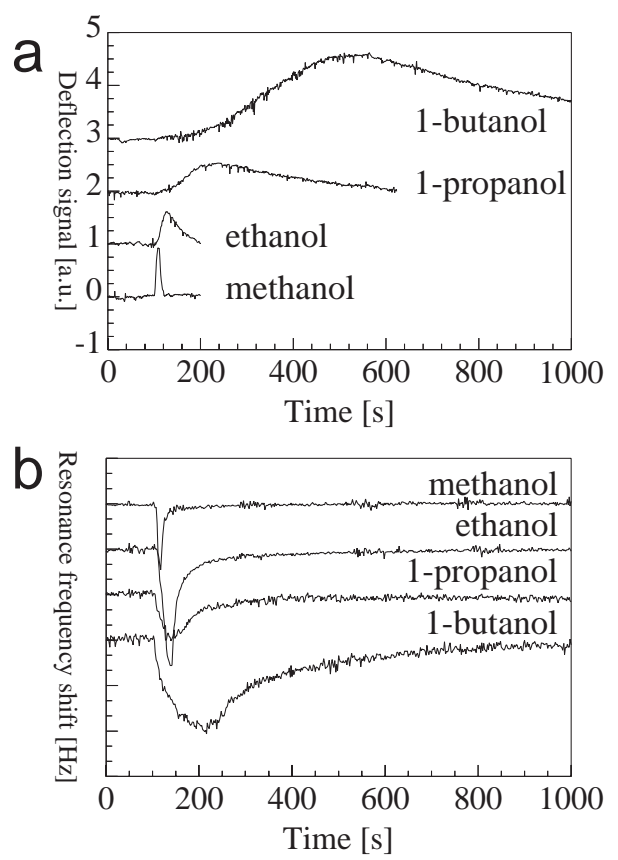

Fig. 4. a Response of a single PMMA-coated sensor in a type B array (raw data) in the static mode upon exposure to $1 \mu \ell$ of methanol, ethanol, 1propanol, and 1-butanol. One unit corresponds to a sensor deflection of $5 \mathrm{~nm}$. b Response of a PMMA-coated sensor to the exposure to $1 \mu \ell$ of the same alcohols in the dynamic mode. The resonance frequency shift (mass change) is analyzed $\left(f_{\mathrm{i}}=52120 \mathrm{~Hz}\right)$. The interval between two ticks corresponds to a frequency shift of $20 \mathrm{~Hz}$ or $\approx 130 \mathrm{pg}$. The material constants used were $E=1.7 \times 10 \times 10^{11} \mathrm{Nm}^{-2}, v=0.25$

maximum surface stress change derived from Fig. $3 b$ is $0.4 \pm$ $0.1 \mathrm{Nm}^{-1}$, as calculated from (1) using (2).

\subsection{Distinguishing between alcohols in the static and dynamic measuring modes}

As an example of the recognition of various chemicals, the diffusion process of four alcohols into the PMMA coating of a cantilever in a type B array is shown in Fig. 4. The PMMA coating is exposed to the vapor of $1 \mu \ell$ of methanol, ethanol, 1-propanol, or 1-butanol. The pressure during the experiments was $962 \mathrm{mbar}$ and the temperature $23^{\circ} \mathrm{C}$. The measurement chamber is open to the environment to allow complete evaporation of the analyte. No washing process is required because the experiment is reversible. No sensitivity change or degradation was observed after 50 cycles of methanol exposure. On exposition to methanol vapor for several hours, the sensor is saturated, but recovers after the methanol vapor is removed. Other sensors coated with PMMA yield comparable results. Figure 4 a shows the static bending of the sensor due to surface stress change, and Fig. 4b displays the resonance frequency shifts due to mass change according to (3). Note that the signal evolution consists of two sections: first, the diffusion of the analyte into the polymer (increase of mass or decrease of resonance frequency in the dynamic mode; compressive stress in the static mode) and, second, the diffusion process of the analyte out of the polymer after the analyte droplet has completely evaporated (resonance frequency increase or stress relief). The signal magnitude increases with the amount of analyte offered, whereas 
the curve shape (temporal evolution of the signal) does not change. Hence it is possible to study analytes quantitatively and qualitatively, even in mixtures [17].

\section{Conclusions}

In conclusion, the setup described here utilizes a time-multiplexing beam deflection technique to obtain a quasi-simultaneous readout of the bending of eight individual sensors assembled in a micromechanical array. The major advantages of this setup include the capability to perform a differential measurement to extract reaction signals, e.g., in noisy environments. Moreover, the micromechanical nature of the sensors implies short response times owing to the small reaction area and high sensitivity. The device provides a cheap, off-the-shelf solution using standard components. The pitch of the fiber ribbon $(250 \mu \mathrm{m})$ enables self-alignment of the light beams on the sensor array and facilitates operation. Currently, eight sensors are being read out quasi-simultaneously, but there is no inherent principle limitation to the number of sensor elements. First applications have demonstrated the capability of detecting $\mathrm{H}_{2}$ and recognizing various alcohols.

Acknowledgements. We thank P. Guéret and H. Rohrer for helpful discussions as well as for their support. We are grateful to R. Brönnimann for preparation of the cantilever coatings. This work is partially funded by the Swiss National Science Foundation priority program MINAST (Micro- and Nanostructure System Technology), Project 7.04 NOSE and the program NFP 36 (Nano-Science).

\section{References}

1. S.C. Minne, S.R. Manalis, C.F. Quate: Appl. Phys. Lett. 67, 3918 (1995)

2. M.I. Lutwyche, Y. Wada: Sensors and Actuators A 48, 127 (1995)

3. S.A. Miller, K.L. Turner, N.C. MacDonald: In Proc. Transducers '97, International Conference on Solid-State Sensors and Actuators, Chicago, June 16-19, 1997 (IEEE, Piscataway 1997) p. 455

4. S.C. Minne, S.R. Manalis, A. Atalar, C.F. Quate: J. Vac. Sci. Technol. B 14, 2456 (1996)

5. J.K. Gimzewski, Ch. Gerber, E. Meyer, R.R. Schlittler: Chem. Phys. Lett. 217, 589 (1994)

6. R. Berger, Ch. Gerber, J.K. Gimzewski, E. Meyer, H.-J. Güntherodt: Appl. Phys. Lett. 69, 40 (1996)

7. J.R. Barnes, R.J. Stephenson, M.E. Welland, Ch. Gerber, J.K. Gimzewski: Nature 372, 79 (1994)

8. G.Y. Chen, T. Thundat, E.A. Wachter, R.J. Warmack: J. Appl. Phys. 77, 3618 (1995)

9. R. Berger, Ch. Gerber, H.P. Lang, J.K. Gimzewski: Microelectronic Engineering 35, 373 (1997)

10. R. Berger, E. Delamarche, H.P. Lang, Ch. Gerber, J.K. Gimzewski, E. Meyer, H.-J. Güntherodt: Science 276, 2021 (1997)

11. T. Thundat, S.L. Sharp, W.G. Fisher, R.J. Warmack, E.A. Wachter: Appl. Phys. Lett. 66, 1563 (1995)

12. W. Göpel, J. Hesse, J.N. Zemel (Eds.): Sensors: A Comprehensive Survey, Vols. 1-9 (VCH, Weinheim 1989-1996)

13. H. Baltes, W. Göpel, J. Hesse (Eds.): Sensors Update, (VCH, Weinheim, Basel 1996)

14. G. G. Stoney: Proc. R. Soc. London, Ser. A 82, 172 (1909)

15. T. Thundat, E.A. Wachter, S.L. Sharp, R.J. Warmack: Appl. Phys. Lett. 66, 1695 (1995)

16. F.C. Tompkins: In Chemisorption of Gases on Metals (Academic Press, London, New York, San Francisco 1978) pp. 200-202

17. H.P. Lang et al.: in preparation 